\title{
Adaptation with the University Environment by Syrian Refugee Students in Zarqa University
}

Nida'a Mohammed Ahmed Khalil

Kindergarten Department - Educational Sciences

Zarqa University - Jordan

salemmaloul@yahoo.com

\author{
Ahmed Issa Daoud \\ Class Teacher - Educational Sciences \\ Zarqa University - Jordan \\ daoudahmed82@yahoo.com
}

Received 27/10/2019

Accepted 31/12/2019

\begin{abstract}
:
The present study aims at identifying the Adaptation with the University Environment by the Syrian refugee students in Zarqa University, the descriptive survey approach methods is used. The sample of the study consists of (80) Syrian refugee students in Zarqa University. A questionnaire is prepared, and the results of the study showed that there were no statistically significant differences between the average degree of students' adaptation to the university environment according to sex, as well as the absence of statistically significant differences between the average degree of students' adaptation to the university environment according to the college.
\end{abstract}

Keywords: Adaptation, Syrian Refugee Students, the University Environment. 


\section{درجة تكيف الطلبة اللاجئين السوريين مع البيئة الجامعية في جامعة الزرقاء}

\author{
أحمد عبسى داود \\ معلم الصف - كلية العلوم التزبوية \\ جامعة الزرقاء - الأردن \\ daoudahmed82@yahoo.com
}

\author{
نداء محمد أحمد خليل \\ رياض الأطفال - كلية العلوم التربوية \\ جامعة الزرقاء - الأردن \\ salemmaloul@yahoo.com
}

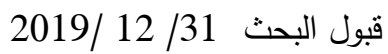

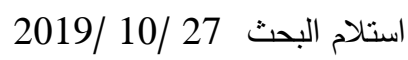

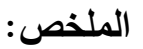

هدفت الدراسة إلى معرفة درجة تكيف الطلبة مع البيئة الجامعية في جامعة الزرقاء، واستخدم في الدراسة المنهج الوصفي المسحي، وتكونت

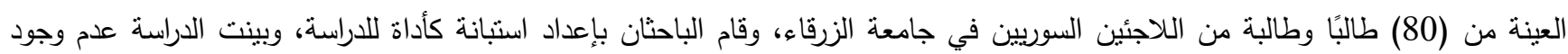

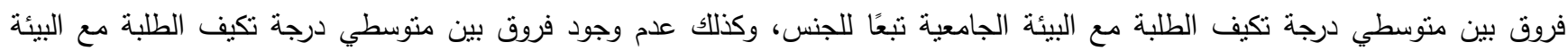

الجامعية تبعًا للكلية.

الكلمات المفتاحية: النكيف، الطلبة السوريون اللاجئون، البيئة الجامعية.

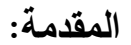

موجهاً نحو إعدادهم لتحمل المسئولية، ومساعدنهم لمواجهة مشكلاتهم، وهذا يمثل الدور الاستراتيجي للجامعة(4).

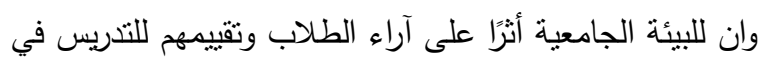

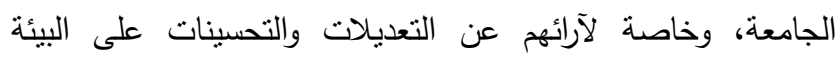

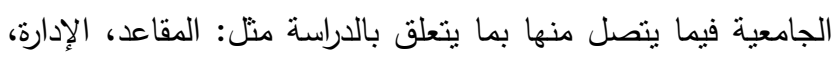
وتتظيم القاعات(13).

وعليه فإن تتمية الدهارات لدى طلبتها، وتعزيز التطور العاطفي والاجتماعي، والخبرة الجامعية، يمكن أن تكون بمثابة طريقة لنقل

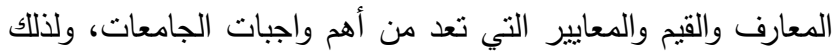

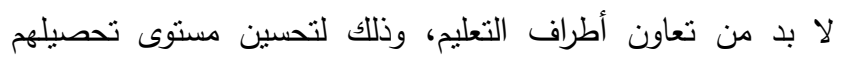

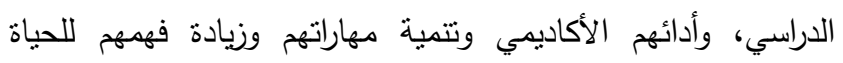
العملية) (8).

وتعتبر الجامعات ذات أهمية كبيرة في مواجهة تحديات التكيف

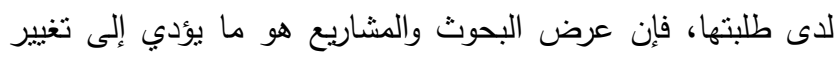

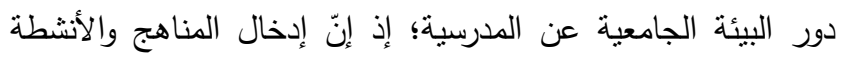

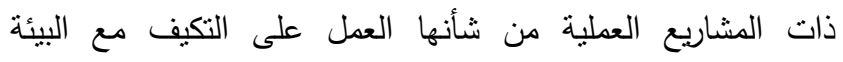

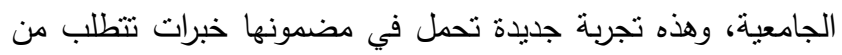

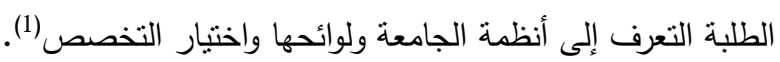
ويعتبر التكيف لدى طلبة الجامعة مفهوما جديدا في تحديد

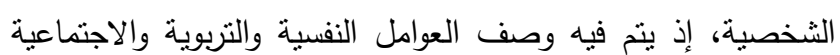

إن التعليم العالي من أهم مقومات التتمية البشرية، فهو يمثل

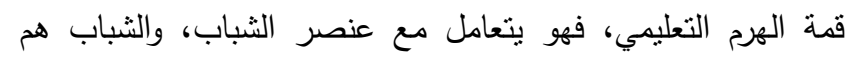

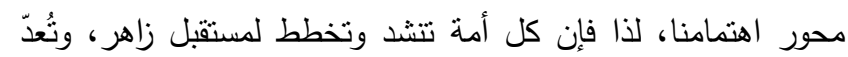
الجامعة من المراحل المهمة في الحياة حيث الاستقلالية واثبات الهوية، وهو أمرٌ هامٌ في المشاركة في الإحساس بالانتماء لجماعة ما، بحيث يكون هذا الفرد جزءً من مجموعة اجتماعية (5). إن الحياة الجامعية لها أهمية لدى الطالب في كل كل مناحي

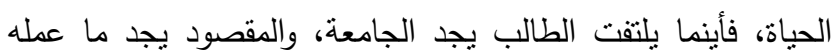
وأنجزه المدرسون في الجامعة ومراكز البحث العلمي، فهذه وسائل الاتصالات، بحيث يوجد اكتثافات العلوم المختلفة، وانجازات الخريجين الذين يقومون بدور القوى البشرية في المجتمع. هذه هي

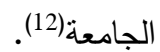

وتشعى المرحلة الجامعية إلى نتمية شخصية الفرد وتهذيب سلوكه مع الأهداف التعليمية من حيث صقل قدراته العقلية، والتدريب

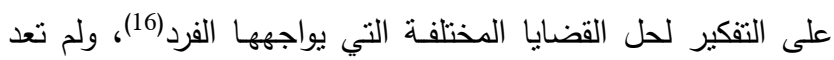
الجامعات مجرد مراكز أكاديمية للبحث العلمي البحت، بحيث يستشعر

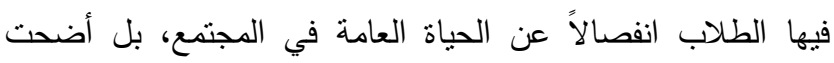
تنظيمات ثقافية للثباب، فالحياة الجامعية ضرب من التفاعل الثقافي

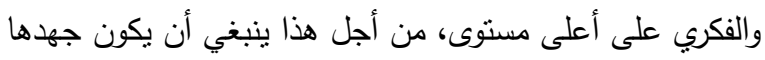


وتبين من النتائج أن طالبات كلية النربية الرياضية في جامعة اليرموك يملكن القدرة على التكيف النفسي والاجتماعي والأكاديمي مع البيئة الجامعية.

دراسة السعايدة والعواودة والحديدي(7) هدفت الى معرفة

مشكلات الطلبة الوافدين من دول الخليج العربي في الجامعات

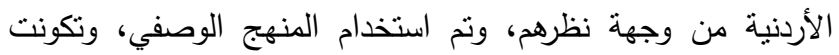

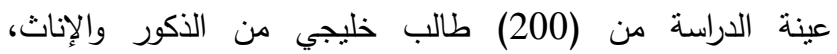
واستخدمت الاستبانة لغايات جمع المعلومات من العينة، وأظهرت نتائج الدراسة أن المشكلات النفسية تحتل المرتبة الأولى عند الطلبة

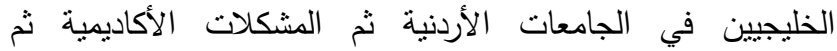

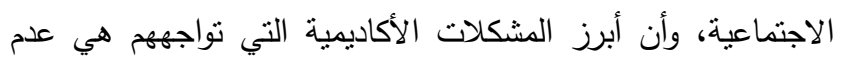

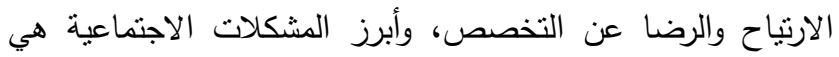

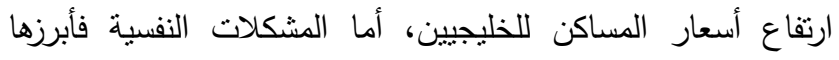
الضيق والاكتئاب نتيجة الابتعاد عن الأسرة.

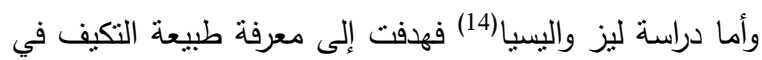

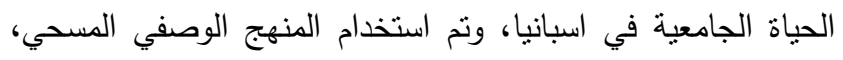

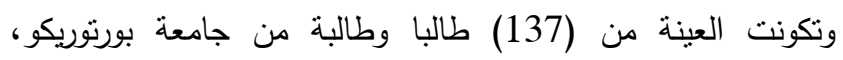
واستخدمت استبانة تتضمن أربعة مجالات فرعية ،هي (التظظيمية،

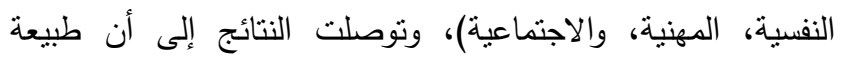
التكيف في الحياة الجامعية كانت بدرجة منوسطة.

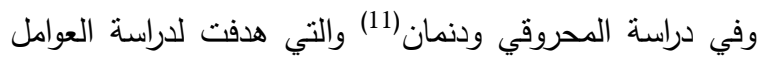
التي تؤثر على تعديل طلاب السنة الأولى في الجامعة الحكومية الوحيدة في سلطنة عمان - جامعة السلطان قابوس، نم استخدام المنهج الوصفي المسحي وتم استخدام استبانة، وتكونت العينة من

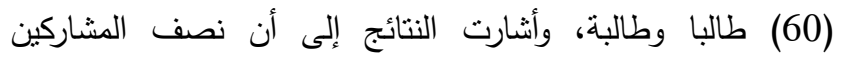
يواجهون صعوبات في التكيف مع بيئتهم الجامعية. دراسة اليكساندر وآخرون (10) والتي هدفت بلى إلى معرفة صعوبات التكيف لطلاب السنة الأولى كأساس لتعزيز تتميتهم الثخصية في

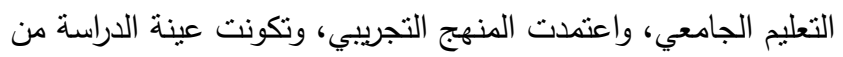
(46) طالبا وطالبة من جامعة نولاليف نولسنوي التربوية في روسيا،

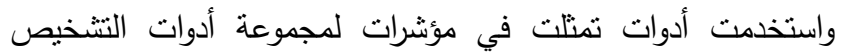
النفسي، وبينت النتائج أن درجة تكيف الطلبة كانت بمستوى منوسط.

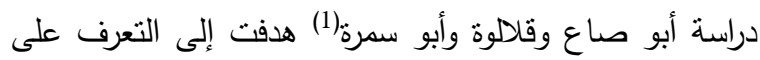

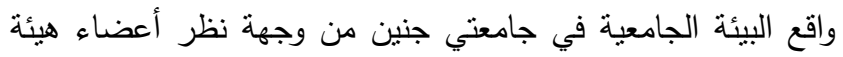

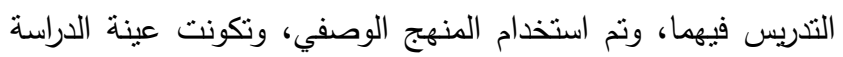
من (170) عضو هيئة تدريس، واستخدمت الاستبانة كأداة للاراسة، وأثنارت نتائج الدراسة إلى أنّ تقديرات أفراد عينة الدراسة لواقع البيئة

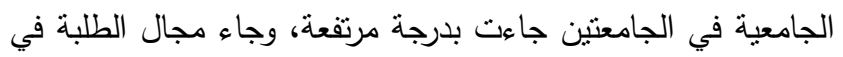

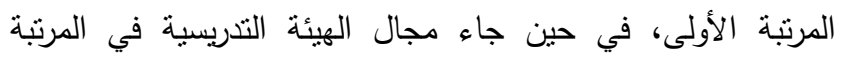
الأخيرة، كما أثنارت النتائج إلى عدم وجود فروق ذات دلالة إحصائية
المؤثرة على تكيف الطلبة، ويتم تحديد المعايير الأساسية لقدرة الطلبة على التكيف في الجامعة، وتعطى الخصائص الموضوعية والثخصية لعدم القدرة على التكيف، أو مؤشرات النجاح للتكيف(15).

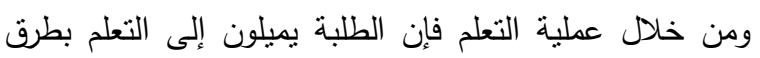
تدريس مختلفة، وأنهم يفضلون استخدام الموارد التعليمية المختلفة التي تسعى إلى مطابقة محتويات التدريس مع أسلوب نعلم الطلبة، وهذا

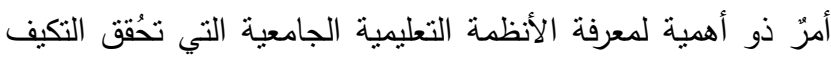
لاى الطلبة، فيصبح الطلبة قادين على التعلم (16).

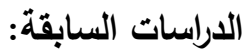

قام الباحثان بالرجوع إلى مختلف الأبحاث التي بينت تكيف

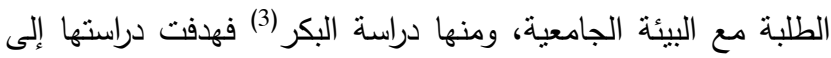
الكثف عن الصعوبات الني تواجه الطالبات المستجدات في الكليات

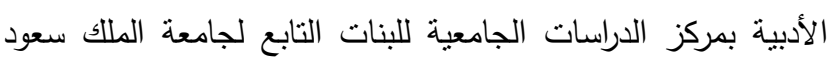

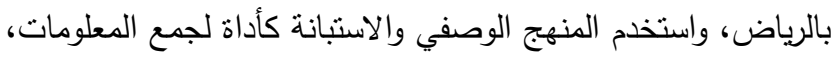

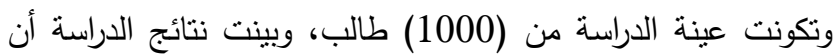

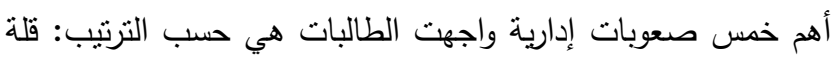

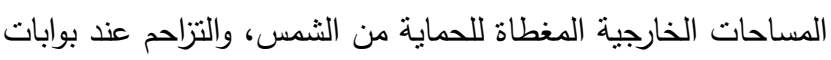

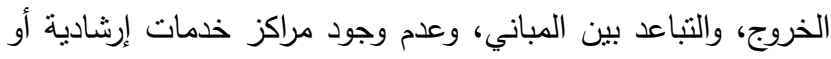
ثقافية أو ترفيهية تتتاسب وأعداد الطالبات، كما أن مساحة الكافيتريات لا نتاسب مع أعداد الطالبات. فيما يتعلق بترنيب الطالبات لأبرز

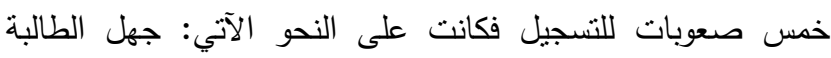

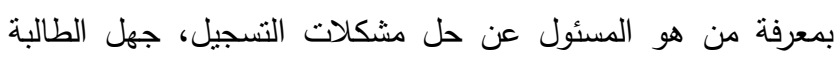
بالأمور الأكاديمية من حيث نسب الغياب والحرمان وأنظمة دخول الامتحان، الازدحام والتكس في أوقات نوزيع إثعارات التسجيل،

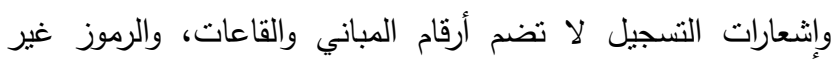

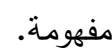
أما دراسة الدمياطي(4) التي هدفت إلى الوقوف على واقع

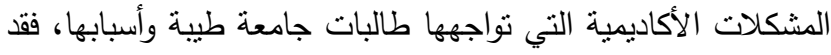
استخدمت الدراسة المنهج الوصفي المسحي، وطبقت استبانه، وتكونت العينة من (384) طالبة، وكثفت نتائج الدراسة أن المشكلات الاتحة

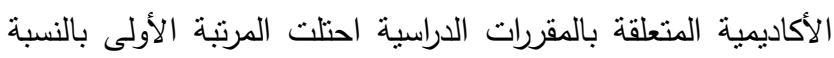

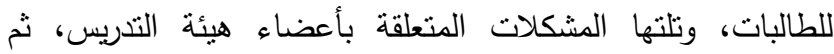

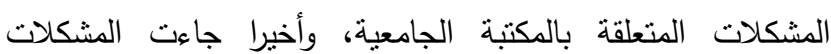

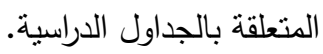

وهدفت دراسة الزيود(5) إلى التعرف على مدى تكيف طالبات

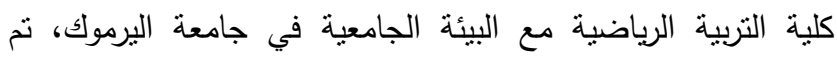
الاعتماد على المنهج الوصفي، وطبقت على عينة بلغت (150)

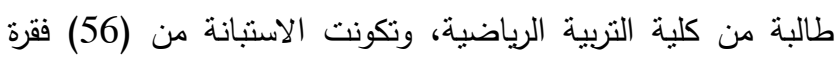

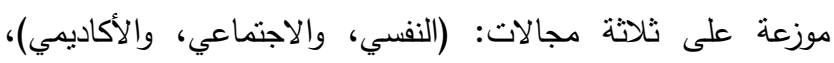




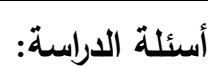

صيغت أسئلة الدراسة على النحو الآتي:

1. ما درجة تكيف الطلبة السوريين اللاجئين مع البيئة البئ

$$
\text { الجامعية في جامعة الزرقاء؟ ماء داء }
$$

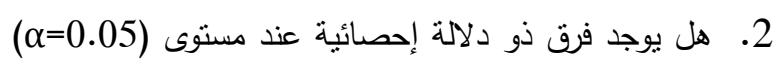

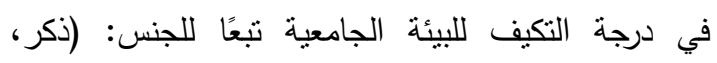

$$
\text { (أنثى) }
$$

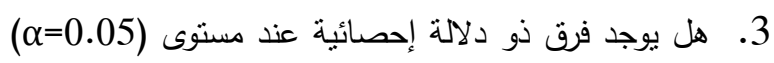

في درجة التكيف للييئة الجامعية تبعًا للكلية: (إنساني،

$$
\text { علمي) }
$$

أهمبة الدراسة:

تنبثت أهمية الدراسة مما يأني:

1. التعرف إلى درجة تكيف الطلبة السوريين اللاجئين في

$$
\text { جامعة الزرقاء مع البيئة الجامعية. }
$$

2. التعرف إلى الفروق في درجة تكيف الطلبة السوريين

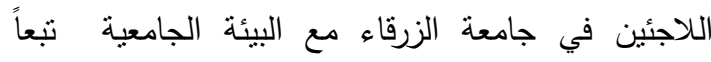

$$
\text { للجنس، والكلية. }
$$

\section{حدود الاراسة ومحدداتها:}

1. الحدود الموضوعية: تقصي درجة تكيف الطلبة السوريين

$$
\text { اللاجئين مع البيئة الجامعية. }
$$

2.

3. الحدود البشرية: الطلبة الحاصلون على الجنسية السورية

$$
\text { وهم اللاجئون في جامعة الزرقاء. }
$$

4. الحدود الزمنية: تم تطبيق هذه الدراسة خلادل الرناء العام الجامعي

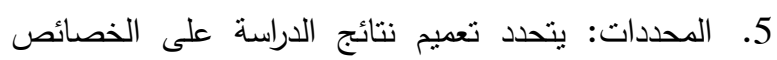
السيكومنرية لأدوات الدراسة: (الصدق، الثنات).

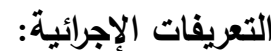

ه التكيف: ثأقلم طلبة جامعة الزرقاء مع العناصر البشرية والمادية والدراسية في البيئة الجامعية.

الطالب اللاجئ السوري: جميع الطلبة الموجودون داخل حرم لئلة الجائل

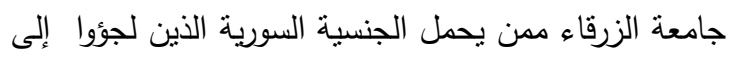

الأردن بسبب تعرضهم للاضطهاد لأسباب مختلفة.

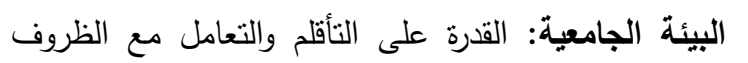
الاجتماعية كالعلاقات والتفاعلات الاجتماعية، ومدى تأقلمه

$$
\text { واندماجه مع قوانين الجامعة. }
$$

بين متوسطات تقديرات أفراد عينة الدراسة لواقع البيئة الجامعية تُعزى

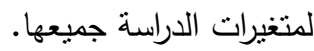
دراسة بريك(2) هدفت إلى معرفة مهارات ما وراء المعرفة

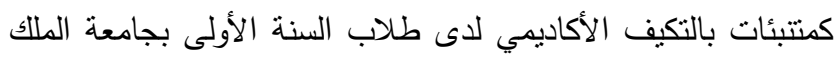

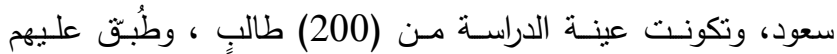

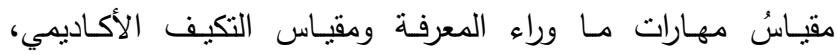
وأظهرت النتائج عدم وجود فروق ذات دلالة إحصائية بين

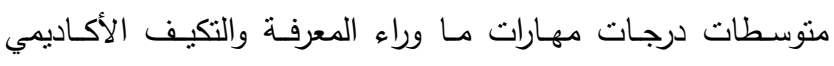

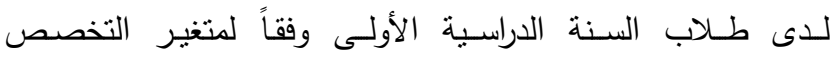

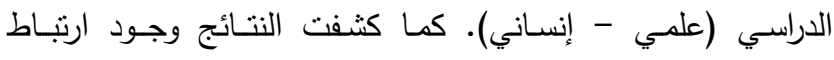

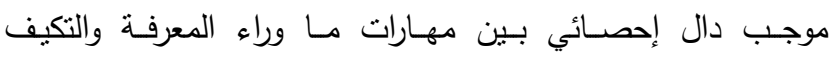
الأكاديمي لدى طلاب السنة الدراسية الأولى.

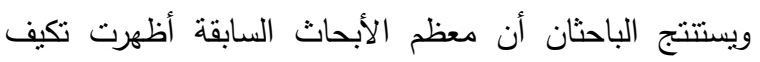
الطلبة مع البيئة الجامعية، وقد استفاد الباحثان منها في تصميم البحث، وفي إعداد الاسنبانه، وقد اختلفت هذه الدراسة عن الدراسات

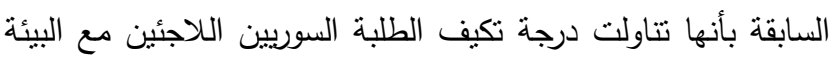
الجامعية في جامعة الزرقاء.

مشكلة الدراسة:

يواجه أكثر الطلبة في الجامعة مشكلات تعمل على إعاقة تكيفهم وتَحدُ من تحقيق نتاجات الجامعة في إعداد الطلبة علميًا والإسهام في نمو شخصياتهم. وذللك لاختلاف الدراسة وطبيعة النظام والقوانين وطبيعة التعامل وأساليب التقويم عن المرحلة الثانوية. وتهدف الجامعة إلى نزويد الطلبة بحزمة من المعارف والمهارات والاتجاهات والقيم، بقدر ما تهذف إلى إيجاد جو تعليمي

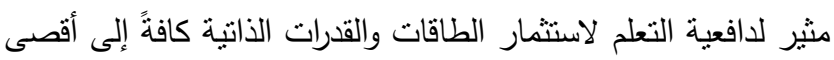

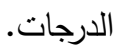
ولاحظ الباحثان أنّ مشكلة الدراسة تكمن في وجود تدنٍ لدى طلبة جامعة الزرقاء في تكيفهم الجامعي، إذ شكل ذلك عبنًا في تتمية الطلبة الثخصية السوية المنزنة والثاملة يقع على البيئة الجامعية،

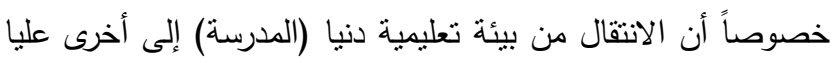

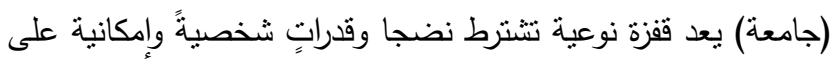
التكيف مع عناصر البيئة الجديدة، إذ يواجه الطلبة فيها عالماً يختلف

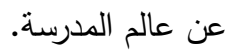
ووفقًا لما سبق فقد ارتأى الباحثان إجراء دراسة تهدف إلى

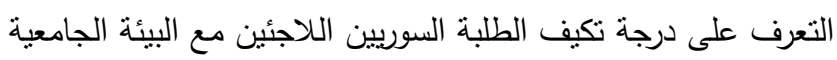
في جامعة الزرقاء. 
من جامعة الزرقاء، وتم حساب الثبات باستخدام كرونباخ ألفا (Cronbach's Alpha)

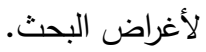

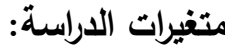

اشتملت الدراسة على المتغيرات الآثية:

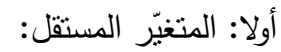

الجنس وله مستويان: (ذكر ، أنثى)

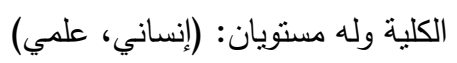

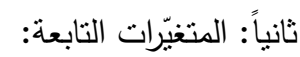

تقديرات الطلبة السوريين اللاجئين مع البيئة الجامعية في الئهيات

$$
\text { جامعة الزرقاء. }
$$

\section{المعالجة الإحصائية:}

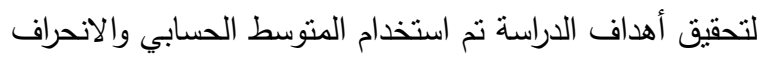

المعياري لعينة الدراسة، كما تم حساب اختبار ( Independent Samples T-Test

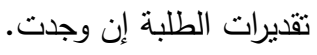

\section{نتائج الدراسة ومناقشتها:}

جرى عرض نتائج الدراسة والإجابة على أسئلتها وفيما يأتي عرضُ لهذه النتائج:

النتائج المتعلقة بالسؤال الأول: ما درجة تكيف الطلبة السوريين اللاجئين مع البيئة الجامعية في جامعة الزرقاء؟

تم حساب المتوسط الحسابي والانحراف المعياري لإجابات أفراد عينة الدراسة عن فقرات أداء الدراسة التي تقبس درجة تكيف الطلبة السوريين اللاجئين مع البيئة الجامعية في جامعة الزرقاء، جدول (2) يبين ذلك.

يظهر من الجدول (2) أن المتوسطات الحسابية لإجابات أفراد عينة الدارسة عن فقرات درجة التكيف تراوحت بين (4.56- 2.1)، وجاءت بالمرتبة الأولى الفقرة (7) "لدي القدرة على التكيف الدراسي في جامعة الزرقاء" بمنوسط حسابي (4.56) ودرجة نكيف مرتفعة،

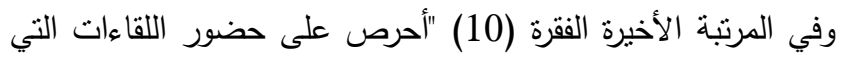
تعلن عنها الجامعة" وبمنوسط حسابي (2.1) وبدرجة تكيف منوسطة،

حيث بلغ المتوسط الحسابي الكلي (3.16) بدرجة تكيف متوسطة. ويعزى ذلك إلى قلة الخدمات الإششادية والنفسية والتوجيهية في الكلية، إذ إن" طلبة السنة الأولى يواجهون تحديًا صعبًا كونها مرحلة الفئة

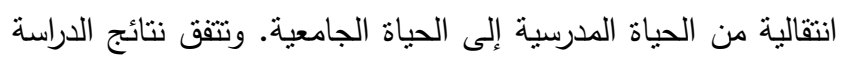
الحالية مـع نتائج دراسة المحروقي ودنمان (11).

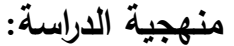

اعتمدت الدراسة المنهج الوصفي المسحي لمعرفة درجة تكيف الطلبة السوريين اللاجئين مع البيئة الجامعية في جامعة الزرقاء.

\section{مجتمع الاراسة وعينتها:}

تكون مجتمع الدراسة من الطلبة السوريين اللاجئين في البيئة الجامعية في جامعة الزرقاء، وتكونت عينة الدراسة من (80) طالبا وطالبة، وكان اختيار العينة بالطريقة القصدية؛ إذ أبدت إدارة الجامعة

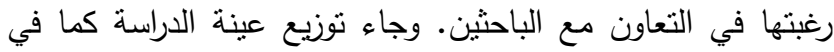

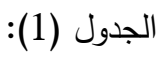

\begin{tabular}{|c|c|c|c|}
\hline النسبة & العدد & فئات المتغير & المتغير \\
\hline 50 & 40 & ذكر & \multirow{3}{*}{ الجنس } \\
\hline 50 & 40 & أنثى & \\
\hline$\% 100$ & 80 & المجموع & \\
\hline 50 & 40 & علمي & \multirow{3}{*}{ الكلية } \\
\hline 50 & 40 & إنساني & \\
\hline$\% 100$ & 80 & المجموع & \\
\hline
\end{tabular}

جدول (1): توزيع عينة الاراسة وفق متغير الجنس والكلية.

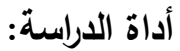

قام الباحثان بإعداد استبانة لجمع البيانات لمعرفة درجة تكيف الطلبة السوريين اللاجئين مع البيئة الجامعية في جامعة الزرقاء، وجرى من خلال الرجوع إلى الأدب النظري والدراسات السابقة الدتعلقة بالتكيف الجامعي كدراسة (البكر (3)؛ والدمياطي(4)). وجرى اعنماد سلم ليكرت تدريج خماسي، حدد بخمسة مستويات هي: (5) بدرجة كبيرة جدًا، (4) بدرجة كبيرة، (3) بدرجة منوسطة، (2) بدرجة قليلة، (1)

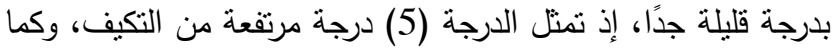
تمثل الدرجة (1) درجة متدنية. وقد حدد الباحثان ثلاثة مستويات

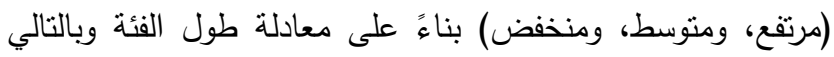
فالمستويات هي منخفض ( أقل من 2.33)، منوسطة (2.34 3.67)، مرتفع (3.68- 5). ووضع الباحثان مجالات وفقرات الاستبانة إذ تكونت الاستبانه في صورتها الأولية من (25) فقرة.

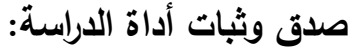

للتحقق من صدق الأداة تم عرضها بصيغتها الأولية على (9) مُحكّمين متخصصين في مناهج وأساليب التنريس، والقياس والتقويم،

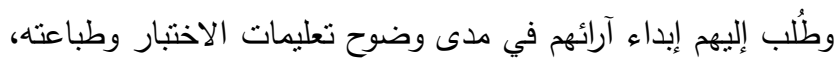
ومناسبة فقراته، وصياغته اللغوية. وفي ضوء آداء آداء المحكين نم تعديل

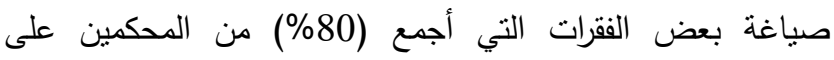
تعديلها. وللتأكد من ثبات الاختبار نم تطبيقه على عينة استطلاعية من خارج البحث وبلغت (20) طالبًا وطالبة من اللاجئين السوريين 
للإجابة عن السؤال جرى استخدام المتوسطات الحسابية

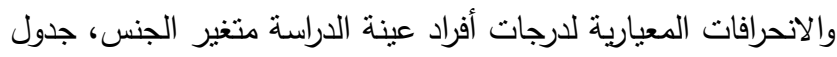
(3) يبين ذلك:

جدول (3): المتوسطات الحسابية والانحرافات المعيارية لارجة التكيف تبعًا لمتغير

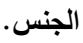

\begin{tabular}{|c|c|c|c|}
\hline الانحراف المعياري & المتوسط الحسابي & العدد & 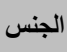 \\
\hline 0.876 & 4.65 & 40 & ذكر \\
\hline 0.548 & 3.39 & 40 & أنثى \\
\hline 0.712 & 4.02 & 80 & الكلي \\
\hline
\end{tabular}

يظهر الجدول (3) المتوسطات الحسابية والانحرافات المعيارية

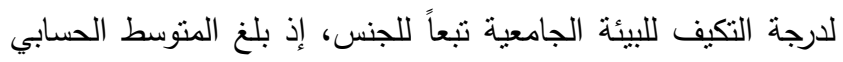
للجنس ذكر (4.65) وبانحراف معياري (0.876). وهو أعلى من لرنابل المنوسط الحسابي للجنس الأنثى الذي بلغ (3.39) وبانحراف معياري

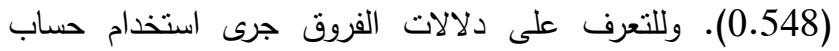
اختبار (Independent Samples T-Test) لارجات أفراد عينة الدراسة عن متغير الجنس، جدول (4) يبين ذلك:

جدول (4): تحليل حساب اختبار (Independent Samples T-Test) لارجة التكيف تبعًا لمتغير الجنس.

\begin{tabular}{|c|c|c|c|c|c|}
\hline الإحصائية & قريمة & متربطات & الحرية & المربعات & مصدر التباين \\
\hline \multirow{3}{*}{0.356} & \multirow{3}{*}{0.807} & 0.365 & 1 & 0.365 & بين الدجموعات \\
\hline & & \multirow{2}{*}{0.452} & 78 & 23.452 & داخل المجموعات \\
\hline & & & 79 & & المجمو \\
\hline
\end{tabular}

يظهر الجدول (4) دلالات الفروق باستخدام تحليل النباين الأحادي، حيث لم يظهر فروق ذات دلالة إحصائية، إذ بلغت قيمة بالاتل (ت) (0.807) وعند مستوى دلالة (0.356) وهي قيم غير دالة إحصائيا. ويمكن تفسير هذه النتيجة بأن الطلبة بعتمدون على أنفسهم في

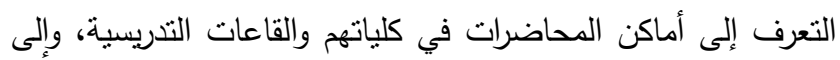
بناء علاقات اجتماعية جديدة مع بعضهم البعض، ووجود فرصة المّات للمناقثة والحوار والتفاعل الاجتماعي مع زملائهم مما أسهم في أسي

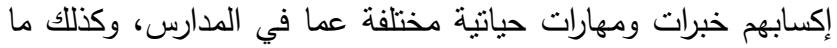
يوفره دليل الطالب الجامعي من توجيهات للطلبة عن الجامعة

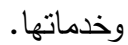
وربما يعود السبب إلى درجة الحرية التي يتمتع الطالب الجامعي مقارنة مع البيئة المدرسية، حيث لا يوجد تقيد بزي معين أو لو لئه طابور صباحي، وهنالك أوقات فراغ بين المحاضرات نُكَّنه من زيارة

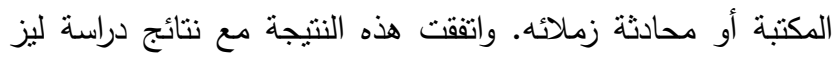
واليسيا(14).
الجدول (2): المتوسطات الحسابية والانحرافات المعيارية لإجابات عينة الدراسة عن الفقرات مرتبة تنازليا.

\begin{tabular}{|c|c|c|c|c|c|}
\hline التكيف & الانحراف & المستوسط & رقم الفقرة & الفقرة & الرتبة \\
\hline 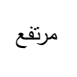 & 0.25 & 4.56 & لدامعة الزرقاء على التكيف الدراسي في & 7 & 1 \\
\hline 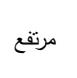 & 0.1 & 4.52 & 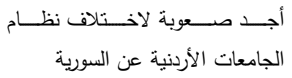 & 13 & 2 \\
\hline مرتفع & 0.3 & 4.38 & تعلمي باللغة الانجليزية عائق التفوقي & 15 & 3 \\
\hline 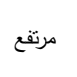 & 0.01 & 4.1 & لدي أهداف محددة من دراستي في & 8 & 4 \\
\hline 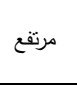 & 0.2 & 3.98 & 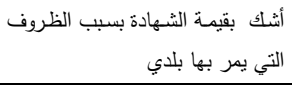 & 1 & 5 \\
\hline 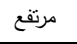 & 0.1 & 3.87 & أنا راضِ عن تخصصي الجامعي & 14 & 6 \\
\hline متوسط & 0.15 & 3.67 & تأفيدني الواجبات الدراسية في إعدادي & 17 & 7 \\
\hline متوسط & 0.41 & 3.45 & 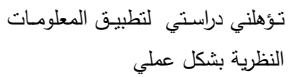 & 21 & 8 \\
\hline متوسط & 0.25 & 3.35 & أسعى لتطوير نفسي أكاديميا & 25 & 9 \\
\hline منوسط & 0.35 & 3.34 & 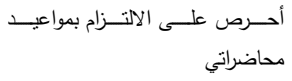 & 19 & 10 \\
\hline متوسط & 0.15 & 3.32 & أحرص على الانتبـاه والمشـاركة في & 24 & 11 \\
\hline منوسط & 0.1 & 3.15 & الاحترام المتبادل مع الزملاء جيدة وقائمة على & 20 & 12 \\
\hline منوسط & 0.1 & 3.1 & أثشارك الطلبة في أنشطتهم & 12 & 13 \\
\hline متوسط & 0.25 & 2.9 & يقدم لي القسم التوجيه التزبوي & 6 & 14 \\
\hline متوسط & 0.14 & 2.89 & بالنسبة لي العام في البيئة الجامعية مريح & 23 & 15 \\
\hline متوسط & 0.13 & 2.87 & 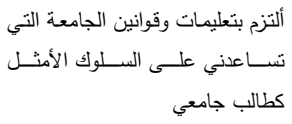 & 9 & 16 \\
\hline متوسط & 0.11 & 2.77 & أنا مرناح لأدائي في الامتحانات & 3 & 17 \\
\hline متوسط & 0.1 & 2.65 & اعمل على تحقيق رؤية كليتي & 5 & 18 \\
\hline متوسط & 0.2 & 2.55 & اعمل على تحقيق رسالة كليتي & 4 & 19 \\
\hline منوسط & 0.35 & 2.43 & تقدم الجامعـة التسـهيلات في إنجـاز & 2 & 20 \\
\hline منوسط & 0.21 & 2.41 & 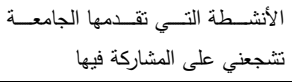 & 11 & 21 \\
\hline ضعيف & 0.14 & 2.3 & المكتبة - الصالة الرياضية مفتوحة لجميع الطلبة: & 18 & 22 \\
\hline ضعيف & 0.23 & 2.3 & منها الطلبة الجامعـة دورات متتوعة يستفيد & 22 & 23 \\
\hline ضعيف & 0.21 & 2.2 & باستــــابع نشـرات وتعميمـات الجامعــة & 16 & 24 \\
\hline ضعيف & 0.17 & 2.1 & تعلن عنه على الجامعة حضور اللقاءات التي & 10 & 25 \\
\hline متوسط & 0.19 & 3.166 & 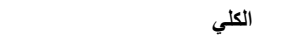 & & \\
\hline
\end{tabular}

النتائج المتعلقة بالسؤال الثاني: هل يوجد فرق ذو دلالة إحصائية عند مستوى (م=0.05) في درجة النكيف للبيئة الجامعية تبعاً للجنس: (ذكر ، أنثى)؟ 


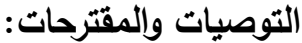

بناء على النتائج التي توصلت إليها الدراسة فإن الباحتَّن

$$
\text { يوصيان بالآتي: }
$$

1. فتح مراكز إرشادية للاجئين السوريين في كافة كليات الجامعة لأهميتها الكبيرة.

2. تأهيل معلمي الجغرافيا وتوعيتهم بمعايير التتمية المستدامة

$$
\text { من خلال الدورات وورش العمل. }
$$

3. الاهتمام بالطلبة اللاجئين السوريين وخاصة في السنة

الأولى لوجود نقلة في المناخ التنظيمي للجامعة.

4. تعبين مرشد نفسي وتربوي داخل الكلية لمساعدة الطلبة

على حل مشكلاتهم التكيفية.

5. إجراء المزيد من الدراسات لتحديد الاحتياجات التربيية

في مجال التتمية المستدامة، والاستفادة من نتائج هذه

$$
\text { الدراسة في هذا المجال. }
$$

\section{References:}

1. Abu Saa, J., Qalalweh, Z., \& Abu Samra, M. (2019). The University Environment in the Palestine Technical University (Khadoori) and the Arab American University from the point of view of their faculty members, The digital repository of the University of Palestine, from: https://scholar.ptuk.edu.ps/handle/12345678 9/368? mode=full.

2. Break, A. (2019). Met cognitive Skills as Predictors of Academic Adjustment Among First Year Students at King Saud University, Jordan Journal of Educational Sciences, 15(1): $65-77$.

3. Al-Baker, F. (2002). Difficulties facing new students in literary colleges at King Saud University and their relationship with their degree of satisfaction with university education, Journal of Educational Sciences, King Saud University, 14(2): 158 $-190$.

4. Al-Domyati, S. (2010). Academic problems of Taibah University students and their relationship to the level of performance - a field study, Higher Education for the Girl.. Dimensions and Aspirations, University of Taiba, 4-6 January: 96 - 140.

5. Al- Zuod, K. (2010). Study some misperceptions of male and female Jordanian students Source, Assiut Journal of Science and Art of Physical Education, 31(1): $239-266$.

6. Al - Sauida, J., Al - Aowda, A., \& Al Hadidi, H. (2015). Arabian Gulf Students Studying in the Jordanian Universities and
النتائج المتعلقة بالسؤال الثالث: هل يوجد فرق ذو دلالة

إحصائية عند مستوى (م=0.05) في درجة التكيف للبيئة الجامعية تبعًا للكلية: (إنساني، علمي)؟

للإجابة عن السؤال جرى استخدام المتوسطات الحسابية

والانحرافات المعيارية لدرجات أفراد عينة الدراسة متغير الكلية، جدول (5) يبين ذلك:

\begin{tabular}{|c|c|c|c|}
\hline الانحراف المعياري & المتوسط الحسابي & العدد & الكلية \\
\hline 0.56 & 3.89 & 40 & علمي \\
\hline 0.43 & 3.88 & 40 & إنساني \\
\hline 0.712 & 4.02 & 80 & الكلي \\
\hline
\end{tabular}

جدول (5): المتوسطات الحسابية والانحرافات المعيارية لارجة التكيف تبعًا لمتغير الكلية.

يظهر الجدول (5) المتوسطات الحسابية والانحرافات المعيارية لارجة التكيف للبيئة الجامعية تبعاً لمتغير الكلية، إذ بلغ المتوسط الحسابي للكلية العلمية (3.89) وبانحراف معياري (0.56). وهو أعلى من المتوسط الحسابي للكلية الإنسانية الذي بلغ (3.88) وبانحراف معياري (0.43). وللتعرف على دلالات الفروق جرى إلى الخى استخدام حساب اختبار (Independent Samples T-Test) لدرجات أفراد عينة الدراسة عن متغير الكلية، جدول (6) يبين ذلك:

\begin{tabular}{|c|c|c|c|c|c|}
\hline الإحصائية & $\begin{array}{l}\text { قيمة } \\
\text { (ت) }\end{array}$ & متوبط المربعات & الحرجية & المربعات & مصدر التباين \\
\hline \multirow{3}{*}{0.374} & \multirow{3}{*}{0.957} & 0.382 & 1 & 0.382 & بين المجموعات \\
\hline & & \multirow{2}{*}{0.399} & 78 & 22.365 & داخل المجموعات \\
\hline & & & 79 & \multicolumn{2}{|c|}{ المجموع } \\
\hline
\end{tabular}
جدول (6): تحليل حساب اختبار (Independent Samples T-Test) لارجة التكيف تبعًا لمتغير الكلية.

يظهر الجدول (6) دلالات الفروق باستخدام تحليل التباين

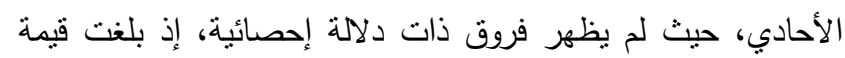
(ت) (0.957) وعند مستوى دلالة (0.374) وهي قيم غير دالة إحصائيا.

ويمكن أن تُعزى هذه النتيجة إلى انسجام الطلبة مع زملائهم في

كلياتهم، والعلاقات الإيجابية مع المدرسين، وكذلك مشاركتهم الفعالة في الأنشطة الجامعية، واعتقادهم بأهمية الدور الذي تقدمه كليتهم من خلال المدرسين من دعم ومساندة للطلبة بشكل عام لصقل شخصياتهم من النواحي كافةً، وتحفيزهم وتشجيعهم وبث الأمل والتفاؤل في دمثي نفوسهم، أدى هذا بدوره إلى الاندماج والانسجام وبالتالي تكيف وانتماء الطلبة مع البيئة الجامعية. وتتقق نتائج الدراسة الحاليـة مـع مـا توصلت إليه نتائج دراسـة الدمياطي (4). 
satisfaction and student evaluation of teaching in the university environment, Academy of Educational Leadership Journal, 14(4): 65-79.

12. Luz, T., and Alicia, G. (2015). University Life Adaptation: Construction and Validation of a Measurement Instrument, International Journal of Educational Excellence, 1(1): $77-92$.

13. Svetlana, T., Tatyana, D., Olga L., Tatyana K., \& Irina M. (2017). Socio-Psychological Adaptation of First Year University Students, Revista ESPACIOS, 38(56): 135 .

14. Velazquez, A., \& Assar, S. (2009). Student Learning Styles Adaptation Method Based on Teaching Strategies and Electronic Media, Educational Technology \& Society, 12(4): 15-29.

15. Walterm L., \& Rafael, L. (2019). University Initiatives in Climate Change Mitigation and Adaptation, Springer International Publishing, Switzerland.
Their Problems from their Point of View, Dirasat: Human and Social Sciences, 42(1): $49-64$.

7. Shamrokh, N., \& Kamal. K. (2011). The social benefits resulting from the physical activity according to the point of view of the Yarmouk University students, An-Najah University Journal for Research: Humanities, 25(5):1337 - 1358.

8. Aleksandr, O., Svetlana, P., Alekse, Y., Tat'yana, P. (2018). A study of frst-year students' adaptation difficulties as the basis to promote their personal development in university education, Psychology in Russia: State of the Art, 11(1): $71-83$.

9. Al-Mahrooqi, R., \& Denman, C. (2015). Adaptation and first-year university students in the Sultanate of Oman, Issues in English education in the Arab world, Publisher: Cambridge Scholars

10. Bitola, E. (2015). Quality Assurance of University Education: Whose

Responsibility?, Asian Journal of Education and Training, 1(1): 8-13.

11. Hill, M., \& Epps, K. (2010). The impact of physical classroom environment on student 